\title{
Guideline Summary for Primary Care Physicians
}

\author{
Werner J. Becker ${ }^{1}$ and Irene Worthington ${ }^{2}$ on behalf of the Canadian Headache \\ Society Acute Migraine Treatment Guideline Development Group
}

Can J Neurol Sci. 2013; 40: Suppl. 3 - S63-S68

\begin{abstract}
Many of the recommendations in this guideline are summarized here to provide a compact source of information for the primary care physician. More detailed discussion can be found in the main guideline document. Tables 2,11A and 11B in Section 3 of the main guideline document list all the acute treatment strategies, and the medications used in each one. Table 9 in Section 3 summarizes medications dosages and side effects. Recommendations related to the efficacy of individual drugs are based on a comprehensive targeted review. Expert consensus statements regarding other aspects of acute migraine treatment are based on a general literature review and expert opinion. All patients for whom acute migraine medications are being prescribed should be educated regarding the common migraine triggers and the important lifestyle factors that may potentially influence their migraine disorder.
\end{abstract}

\section{OBJective}

The primary objective of this guideline is to assist the physician in choosing an appropriate acute medication for an individual with migraine, and to assist the physician in using the chosen medication in the most effective manner.

\section{Goals of acute migraine therapy}

The goal is to render the patient pain free within two hours after treatment. Not all patients can achieve this goal with current acute treatment options, but if a patient is not reaching this goal, or at least able to function reasonably well at two hours, another therapeutic option should be tried if possible.

i. Several acute medication trials may be necessary before an appropriate acute medication is found for a specific patient. Some patients with attacks of varying severity may need access to more than one medication for successful migraine management.

\section{Avoiding medication overuse}

$i$. When initiating treatment with acute migraine medications, the patient should be educated with regard to medication overuse headache. Patients should avoid use of ASA, NSAIDs and acetaminophen on more than 14 days per month, and use of triptans, ergots, opioids, or combination analgesics on more than 9 days a month. Patients taking different acute medications on different days should limit their total use of acute medications to 9 days a month if one of their medications is a triptan, a combination analgesic, an ergotamine, or an opioid.

ii. Patients should be advised to monitor their acute medication use if their attacks are frequent, preferably with a headache diary, in order to reduce the risk of medication overuse headache.

iii.Pharmacological prophylaxis should be considered for patients with frequent migraine attacks that may be at risk of medication overuse.

Which acute migraine medication should be used?

An acute medication should be chosen based on evidence for efficacy, side effect profile, migraine attack severity, presence of co-existing medical or psychiatric disorders, and patient preference. A number of acute medications have evidence for efficacy. Acute medications can be divided into those with a strong recommendation for use, and those with a weak recommendation. A strong recommendation means that the medication could be used for most patients, and that the benefits of therapy outweigh the potential risks (although contraindications still need to be observed). A weak recommendation indicates that the intervention could still be useful, but it would not be appropriate for many patients, often because of potential side effects. With a weak recommendation, the balance between risks and benefits is closer or more uncertain,

MAY BE USED FOR MOST PATIENTS: The following acute medications for migraine received a strong recommendation for use:

1. High quality evidence: Triptans (almotriptan, eletriptan, frovatriptan, naratriptan, rizatriptan, sumatriptan, and zolmitriptan) are recommended for the acute treatment of migraine attacks that are likely to become moderate or severe.

2. High quality evidence: ASA (975-1,000 mg tablets or effervescent formulation), given with oral metoclopramide $(10 \mathrm{mg})$ if nausea is present, is recommended for the acute treatment of migraine attacks of all severities. 
3. High quality evidence: Ibuprofen [400 $\mathrm{mg}$ tablet or solubilized (liquid containing capsules) formulation] is recommended for the acute treatment of migraine attacks of all severities.

4. Moderate quality evidence: Ibuprofen (400 $\mathrm{mg}$ ) in solubilized formulation (liquid containing capsules) is recommended for the acute treatment of migraine attacks of all severities for patients desiring a faster onset of therapeutic effect as compared to the regular ibuprofen tablets.

5. High quality evidence: Naproxen sodium in immediate release formulation (500 or $550 \mathrm{mg}$; up to $825 \mathrm{mg}$, if needed and tolerated) is recommended for the acute treatment of migraine attacks of all severities.

6. High quality evidence: Diclofenac potassium $(50 \mathrm{mg}$ tablet or powder for oral solution) is recommended for the acute treatment of migraine attacks of all severities.

7. Moderate quality evidence: Diclofenac potassium powder for oral solution $(50 \mathrm{mg})$ is recommended for the acute treatment of migraine attacks of all severities for patients desiring a faster onset of therapeutic effect as compared to the diclofenac oral tablet formulation.

8. High quality evidence: Acetaminophen (1,000 mg), alone or in combination with oral metoclopramide $(10 \mathrm{mg})$, is recommended for the acute treatment of mild or moderate migraine attacks.

9. High quality evidence: If migraine response to sumatriptan is inadequate, consider use of naproxen sodium $500 \mathrm{mg}$ to be given simultaneously with the triptan.

10.Low quality evidence: If migraine response to other triptans (other than sumatriptan) is inadequate, consider the addition of an NSAID (e.g., naproxen sodium) to be given simultaneously with the triptan.

MAY BE USED FOR SOME PATIENTS: The following acute medications for migraine received a weak recommendation for use:

1. Moderate quality evidence: Dihydroergotamine (intranasal or subcutaneous self-injection) may be considered for the acute treatment of moderate or severe migraine attacks.

2. Moderate quality evidence: (Not for routine use) Ergotamine may be considered for use is some patients, for example when triptans are not available to the patient or not effective.

3. Moderate quality evidence: (Not for routine use) Tramadol in combination with acetaminophen may be considered for patients with moderate or severe migraine attacks when triptans and/or NSAIDs are ineffective or contraindicated and for occasional use as rescue medication when the patient's regular medication has failed. Frequency of use should be closely monitored, preferably with use of headache diaries.

4. Low quality evidence: (Not for routine use) Codeinecontaining combination analgesics may be considered for patients with moderate or severe migraine attacks when triptan and/or NSAIDs are ineffective or contraindicated and for occasional use as rescue medication when the patient's regular medication has failed. Frequency of use should be closely monitored, preferably with use of headache diaries.

DON'T USE ROUTINELY: The following medications received a strong recommendation that they should NOT to be used routinely for the acute treatment of migraine

1. Moderate quality evidence: Ergotamine should not be used routinely for acute migraine attacks, due to inferior efficacy compared to the triptans, and because of the potential for more side effects.

2. Low quality evidence: Oral opioids, including codeine, are not recommended for routine use in migraine, due to lack of evidence for superiority to standard drugs (NSAIDs and triptans), and the risk of dependencelabuse, potential for development of medication overuse headache, and the possibility of a withdrawal syndrome following discontinuation.

3. Low quality evidence: Tramadol alone or in combination with acetaminophen is not recommended for routine use in migraine, due to lack of evidence for superiority to standard drugs (NSAIDs and triptans), and the risk of dependencelabuse, potential for development of medication overuse headache, and the possibility of a withdrawal syndrome following discontinuation.

DON'T USE: The following medications received a strong recommendation that they should NOT be used for acute migraine therapy (except perhaps under very exceptional circumstances):

1. Low quality evidence: Butorphanol nasal spray, although effective for acute migraine, should be avoided (except in exceptional circumstances) for the acute treatment of migraine, due to lack of evidence for superiority to standard drugs (NSAIDs and / or triptans), risk of dependencelabuse, potential for development of medication overuse headache, and the possibility of a withdrawal syndrome following discontinuation. When used, frequency of use should be closely monitored, preferably with use of headache diaries.

2. Low quality evidence: Barbiturate (i.e., butalbital)containing combination analgesics should be avoided (except in exceptional circumstances) for the acute treatment of migraine, due to lack of evidence for superiority to standard drugs (NSAIDs and / or triptans), risk of dependencelabuse, potential for development of medication overuse headache, and the possibility of a withdrawal syndrome following discontinuation of high doses.

The following anti-emetics received a strong recommendation for use in conjunction with acute migraine medications (there is inadequate evidence to make a recommendation for the use of dimenhydrinate or ondansetron):

1. Moderate quality evidence: Metoclopramide $(10 \mathrm{mg}$ orally) is recommended for use with acute migraine medications for migraine attacks to improve relief of nausea. 
2. Low quality evidence: Domperidone (10 $\mathrm{mg}$ orally) is recommended for use with acute migraine medications for migraine attacks to improve relief of nausea.

Based on evidence from randomized controlled trials, metaanalyses, and systematic reviews, the following additional strong recommendations were made for acute migraine therapy:

\section{Try another triptan if the patient response is not excellent:}

1. Moderate quality evidence: If a patient does not respond well to one triptan or tolerates it poorly, other triptans should be tried over time in subsequent attacks. It is recommended that patients wait 24 hours before trying another triptan.

\section{Treat early if possible:}

2. High quality evidence: Patients with migraine attacks that usually peak at moderate or severe intensity should be advised to take triptans early during their migraine attacks while pain intensity is still mild (caution the patient regarding medication overuse headache).

\section{Approach to the individual patient}

An acute medication should be chosen for a specific patient based on migraine attack severity and on the patient history of response to previously tried acute medications. The following expert consensus statements outline such an approach.

$i$. Patients with severe attacks that often require bed rest: a. Should be given a triptan (with an anti-nauseant, if necessary), consistent with the stratified treatment approach.

b. Subcutaneous sumatriptan $6 \mathrm{mg}$ may be the preferred triptan for severe attacks with early vomiting, or for severe attacks which do not respond to other triptan formulations.

ii. Patients with less severe attacks and who have not had adequate trials of non triptans:

a. Should be educated about acute treatment options.

b. An anti-emetic (metoclopramide $10 \mathrm{mg}$ or domperidone

$10 \mathrm{mg}$ ) can be added to acute migraine medications if needed for nausea.

c. A "step care across attacks" strategy as outlined below can be initiated with careful patient follow up.

Step 1: ASA 1,000 mg, ibuprofen $400 \mathrm{mg}$, diclofenac potassium $50 \mathrm{mg}$, naproxen sodium $550 \mathrm{mg}$, or acetaminophen $1,000 \mathrm{mg}$ if NSAID intolerant. For patients with relatively severe attacks (but not usually requiring bed rest), a triptan can be prescribed at the same time. The triptan can be used as a rescue medication by the patient as necessary if the NSAID or acetaminophen occasionally fails, or can be adopted as the patient's primary acute migraine medication if the NSAID or acetaminophen proves unhelpful (see step 2 below).

Step 2: For patients not responding well to NSAIDs, use a triptan as the primary medication for acute migraine therapy:

a. At least three different triptans should be tried (in different attacks) if the response to the first triptan is not excellent.
An excellent response is defined as pain free or almost pain free with the ability to resume usual activities at $2 \mathrm{~h}$ post-dose, and no significant side effects

b. A triptan should be used to treat approximately three separate migraine attacks before being judged effective or ineffective.

c. Intranasal triptans which are partially absorbed through the nasal mucosa (e.g., zolmitriptan $5 \mathrm{mg}$ ) may be preferred to oral triptans for patients with nausea.

d. Orally dissolving tablets (wafers) may be the preferred oral triptan for patients with nausea exacerbated by taking fluids.

$e$. For patients with more than one migraine attack severity, providing medications from two different classes should be considered (e.g., a triptan and NSAID).

Step 3: For patients whose response to triptans remains inadequate because of incomplete relief or frequent treatment failure, an NSAID (e.g., naproxen sodium 500 - $550 \mathrm{mg}$ ) should be used simultaneously with their triptan.

Step 4: For patients with a good response to their triptanNSAID combination therapy but who experience occasional treatment failure, consider the need for a rescue medication. Rescue medications can include additional NSAIDs (oral, rectal, or injectable with oral metoclopramide), prochlorperazine (oral, rectal), corticosteroids, and acetaminophen with tramadol or codeine (not for routine use; monitor frequency of use carefully).

Step 5: For patients who do not respond satisfactorily to an NSAID-triptan combination, the use of dihydroergotamine (nasal spray or self-injection), combined with oral metoclopramide (if needed), can be considered.

Step 6: Although not recommended for routine use in migraine, opioid analgesics (e.g., acetaminophen with codeine or tramadol) remain an option for patients without a satisfactory response to earlier treatment steps, but:

a. their frequency of use should be closely monitored (using a headache diary)

b. behavioural and pharmacological preventive treatment options should be explored

c. these medications are also a treatment option for patients with contraindications to vasoconstrictor drugs and who do not respond to NSAIDs

\section{Acute treatment strategies to guide acute medication choice}

There are many drugs available for acute migraine treatment. These need to be chosen based upon patient clinical characteristics, and each needs to be used appropriately. The medications are organized here into a number of treatment strategies. Once the clinical data on a specific patient has been gathered, including attack severity and past medication use and response, an appropriate strategy can be chosen and implemented. Depending upon the patient's response to the chosen pharmacological treatment strategy, the same strategy can be continued, or a new strategy can be implemented.

The primary drugs for acute migraine attack treatment are the NSAIDs (including ASA) and the triptans. Acetaminophen is widely used, but is considered less effective than the NSAIDs, and is suitable mainly for attacks of mild to moderate severity. Metoclopramide is recommended when an anti-nauseant is needed, as more evidence is available for efficacy for this drug 
than for the related medication, domperidone. Domperidone may have fewer side effects; however, domperidone may be associated with QT prolongation in some patients.

1. Mild to moderate attack strategies: These are for patients with attacks that are not disabling (i.e., attacks do not require bed rest, and do not stop participation in activities, although it may be somewhat difficult for the patient to continue).

\section{a. Acetaminophen strategy:}

$i$. Acetaminophen is an effective option for acute migraine therapy for some patients with attacks of mild to moderate intensity.

\section{b. NSAID strategy:}

i. NSAIDs (including ASA) are helpful for many patients with migraine. Although it cannot be predicted which NSAID will be best for a specific patient, pharmacokinetic differences between them should be considered when treatment recommendations are made.

ii. For patients with migraine attacks that increase in intensity rapidly, diclofenac potassium powder for oral solution, effervescent ASA, and solubilized ibuprofen capsules have a rapid onset of action and may be particularly helpful.

iii.For patients with migraine attacks that increase in intensity rapidly, diclofenac potassium tablets have the most rapid onset of action for tablet formulations of NSAIDs (note: diclofenac potassium powder for oral solution has a more rapid oral absorption than tablets).

$i v$. The long plasma half-life of naproxen sodium may make it particularly helpful for patients with prolonged migraine attacks.

\section{Moderate-severe attack or NSAID failure strategies}

\section{a. NSAID with triptan rescue strategy:}

$i$. For patients with relatively severe attacks (but not usually requiring bed rest), an NSAID can be tried (if not tried and failed previously), and a triptan can be prescribed at the same time. The triptan can be used as a rescue medication by the patient as necessary if the NSAID occasionally fails, or can be adopted as the patient's primary acute migraine medication if the NSAID proves unhelpful.

\section{b. Triptan strategy:}

$i$. It should be recognized that the response of an individual patient to a specific triptan cannot be predicted with accuracy. Patients with a less than optimal response to their current triptan should be encouraged to try several other triptans in different migraine attacks to determine if they will obtain better relief.

ii. Patients should be encouraged to take their triptan early in their attacks while pain is still mild, although caution may need to be exercised in patients with frequent attacks to avoid medication overuse.
iii.For severe migraine attacks with early vomiting, the use of subcutaneous sumatriptan $6 \mathrm{mg}$ should be considered. Zolmitriptan nasal spray $5 \mathrm{mg}$ may be an alternative choice for some patients. These formulations should also be considered for all patients with severe nausea, particularly those who have nausea early in their attacks, and for attacks not responsive to oral triptan medications.

iv. For patients with moderate or severe migraine attacks who require triptan therapy, and whose attacks build up rapidly in intensity, rizatriptan $10 \mathrm{mg}$ tablets, eletriptan $40 \mathrm{mg}$ tablets, zolmitriptan $5 \mathrm{mg}$ nasal spray, and sumatriptan $6 \mathrm{mg}$ SC injection should be considered.

v. For patients with moderate or severe attacks who experience side effects on other triptans, almotriptan $12.5 \mathrm{mg}$ tablets should be considered.

vi.For patients who experience frequent headache recurrence on triptan therapy, the use of eletriptan $40 \mathrm{mg}$ or frovatriptan $2.5 \mathrm{mg}$ tablets should be considered, or the addition of naproxen sodium to the patient's current triptan.

vii.For patients with nausea or vomiting who require an additional anti-emetic, metoclopramide, domperidone, or if necessary, prochlorperazine can be considered, to be taken with the triptan or triptan-NSAID combination.

For patients on the triptan strategy with headache recurrence within 24 hours after successful acute treatment:

$i$. When patients experience recurrence of a migraine headache attack after initial headache relief from a triptan, a second dose of the triptan should be recommended.

ii. For patients who experience frequent headache recurrence on triptan therapy, the use of eletriptan, frovatriptan, or dihydroergotamine (DHE) should be considered instead of the patient's current triptan, or the addition of naproxen sodium to the patient's current triptan.

For patients on the triptan strategy who have occasional treatment failure (but not often enough to move on to the triptanNSAID combined strategy):

$i$. When patients experience occasional triptan failure with headache persistence two hours after taking a triptan, a rescue medication from another drug class should be considered, as opposed to dosing again with their triptan.

\section{Timing of triptan use in patients with migraine with aura:}

i. Patients with migraine with aura should be advised to take their triptan at the onset of the pain phase, although triptan treatment during typical migraine aura is safe, and if patients find that treatment during the aura is effective, there is no reason to discourage this practice.

\section{Refractory migraine strategies}

These are patients who have not responded satisfactorily to NSAIDs and / or triptans. 


\section{a. Triptan-NSAID combination strategy:}

i. For patients whose response to triptans alone is inadequate, an NSAID (e.g., naproxen sodium 500 - 550 mg) should be used simultaneously with their triptan.

ii. For patients with nausea, or where poor drug absorption is suspected, oral metoclopramide $10 \mathrm{mg}$ or domperidone $10 \mathrm{mg}$ can be given with the triptan.

\section{b. Triptan-NSAID combination with rescue medication} strategy:

i. For patients with severe migraine attacks where their triptan or triptan-NSAID combination occasionally fails to provide adequate relief, a rescue plan should be discussed with the patient. This may include a rescue medication to be taken at home when their usual medication fails.

ii. In providing a rescue medication, the patient needs to be carefully assessed, and the medication tailored as much as possible to the patient's needs. For parenteral formulations, careful patient training is essential, and consideration should be given as to whether the patient can safely administer the medication.

iii.For many rescue medications, in particular opioids and dexamethasone, frequency of use should be carefully monitored to ensure patient safety, and in the case of opioids to avoid medication overuse headache, abuse, dependence and possible addiction.

iv. Rescue medications that can be considered, either alone or in combination, include:

a. NSAIDs with or without an anti-emetic, including ketorolac $60 \mathrm{mg}$ by IM self-injection and rectal indomethacin

b. Dopamine antagonists including prochlorperazine suppositories

c. Oral dexamethasone or another steroid, either as a single dose or a short steroid taper over several days

d. Tramadol or codeine-containing combination analgesics (limit use to not more than nine days a month) $e$. Other opioids (suggest limiting use to not more than seven days per month)

v. Migraine attack preventive management options, both pharmacological and behavioural, should be considered for all patients where acute therapy is not adequately successful or the patient is at risk of medication overuse headache.

\section{c. Dihydroergotamine strategy:}

i. Dihydroergotamine (DHE) by nasal spray [one spray $(0.5 \mathrm{mg})$ in each nostril, repeated once after 15 - 30 minutes; maximum daily dose eight sprays) or selfinjection (0.5 - $1 \mathrm{mg}$; maximum daily dose $3 \mathrm{mg}$ ) is an option for acute migraine therapy for patients who do not respond well to triptan-NSAID combination therapy (but not as a rescue therapy as it is also a vasoconstrictor).

ii. DHE self-injection (SC or IM) requires individual patient training in safe injection techniques, but provides more reliable drug absorption than the intranasal route.

\section{Vasoconstrictor unresponsive or contraindicated strategy:}

$i$. For patients with contraindications to vasoconstrictors or who have proven unresponsive to vasoconstrictors (triptans, DHE, and / or ergotamine), acetaminophen, NSAIDs (including ASA), acetaminophen-NSAID-caffeine combinations (not available in Canada as combination products), dopamine antagonists (e.g., prochlorperazine), occasional steroid use, and opioid-containing combination analgesics can be considered.

ii. Consideration needs to be given to the safety of NSAIDs in patients with cardiovascular disease. Because of a relatively benign cardiovascular profile, naproxen sodium may be the NSAID of choice, if effective, for patients with cardiovascular disease, particularly in patients who require relatively frequent use.

iii.If use of tramadol or codeine-containing combination analgesics is necessary, frequency of use should be carefully monitored and limited to use on 9 days a month or less.

iv. If, in exceptional cases, use of strong opioids or barbiturate-containing analgesics is considered, their frequency of use should be carefully monitored to avoid medication overuse headache, dependence, abuse, and possible addiction. Suggest limiting use to not more than seven days per month.

v. Behavioural treatment strategies and pharmacological prophylaxis may need to be maximized if a satisfactory pharmacological acute treatment cannot be established.

\section{Special acute treatment strategies}

\section{Menstrual migraine strategy:}

$i$. In most patients, acute treatment of menstrual migraine attacks is similar to acute treatment of attacks occurring at other times during the menstrual cycle.

ii. For patients with refractory menstrual migraine who have a sufficient migraine attack frequency to justify general prophylactic therapy, this may be the best option.

iii. For selected patients with refractory menstrual migraine with predicable timing of menstrual cycles, short-term monthly prophylaxis can be considered. Among the available options (frovatriptan, zolmitriptan, naratriptan, and naproxen), frovatriptan $2.5 \mathrm{mg}$ twice a day starting two days before menstruation onset and continuing for six days has the strongest evidence for efficacy.

$i v$. In selected patients, hormonal manipulation including estrogen supplementation around the time of menstruation, and continuous use of combination oral contraceptives can be considered but other treatment options should be tried first. If continuous use of combined oral contraceptives is being considered, contraindications and cautions for these (e.g., smoking, migraine aura, etc) should be observed (see discussion with regard to migraine with aura in Section 3, main guideline document).

\section{Migraine during pregnancy strategy:}

$i$. Avoid use of medications during pregnancy if possible, especially during the first trimester, and consider use of 
non-pharmacologic strategies (e.g., trigger avoidance, relaxation exercises, etc).

ii. Acetaminophen is generally regarded as the safest analgesic for use during pregnancy.

iii. Alternatives to acetaminophen when acetaminophen is not adequate that may be considered for use during pregnancy include acetaminophen plus codeine combination products (intermittent use).

$i v$. Sumatriptan is also a potential option for acute migraine treatment in pregnancy, but is not recommended for routine use. There is significant evidence that the risks of sumatriptan use in pregnancy are minimal. It may be considered when migraine headaches are severe with significant disability and/or vomiting, other medications have failed during similar attacks, and the benefits appear to outweigh potential risks. There is much less information available regarding the safety of the other triptans during pregnancy; therefore, they should be avoided.

v. NSAIDs (e.g., ibuprofen, naproxen sodium) should be used with caution during pregnancy (possible increased risk of spontaneous abortion in first trimester), and should be discontinued before week 32 .

$v i$. Because of the long-lasting effects of ASA on platelet function, other NSAIDs are preferred to ASA for use during pregnancy.

vii.Metoclopramide has not been associated with birth defects, and may be used during pregnancy. Dimenhydrinate is considered relatively safe for use as an anti-emetic during pregnancy (but there is no controlled trial evidence for efficacy in migraine). Domperidone should be avoided, as there is a lack of data with regard to its use during pregnancy.

viii. Ergot alkaloids are contraindicated during pregnancy.

\section{Migraine during lactation strategy:}

i. Acetaminophen is considered safe during lactation.

ii. Ibuprofen is the NSAID of choice during breast feeding. Diclofenac, ketorolac, and naproxen are also considered compatible with breast feeding, but with less data. ASA should be avoided. iii. Sumatriptan is considered compatible with breast feeding . iv. Metoclopramide, domperidone, dimenhydrinate, and prochlorperazine are all considered safe in breastfeeding.

v. If pain medication is necessary in a breastfeeding mother, the safest drugs are acetaminophen and the NSAIDs. If opioids are considered necessary:

a. Morphine is considered the opioid of choice if potent analgesia is required in breastfeeding mothers (however, there is lack of evidnce for efficacy of oral morphine in migraine).

b. Codeine in occasional doses is considered generally safe, although serious toxicity has been reported in maternal ultra-fast metabolizers (caution if premature infant or neonate less than 4 weeks old).

c. Avoid codeine for long-term therapy because of its variable maternal metabolism, because multiple cases of neonatal toxicity have been reported, and more effective opioid choices are available.

d. Avoid high doses of opioids in breastfeeding women.

e. For all opioids, exercise particular caution if the breastfeeding infant is under one month old.

\section{Conclusion}

There are many options for acute migraine therapy, but the NSAIDs (including ASA) and the triptans are the primary medications for acute migraine treatment, with or without the addition of an anti-emetic such as metoclopramide or domperidone. For patients with severe attacks which often render them bed ridden, it is important to try a triptan quickly to increase the likelihood of obtaining a good therapeutic response. For patients with attacks of lesser severity who have not tried NSAIDs, it is recommended that these be tried first. It is important, though, to inform patients that other options are available to prevent patients from becoming discouraged lapsed consulters who feels that nothing more can be done for them. Acute migraine treatment needs to be individualized for each patient, and consideration of appropriate formulation/route of administration is important. 